\title{
Transferencia de recursos en el ámbito internacional $^{*}$
}

\author{
CAMBIOS ESTRUCTURALES IN EL MUNDO MODERNO: UN NUEVO \\ RUMBO EN I.A HISTORIA DE LAS RELACIONES ECONÓMICAS INTERNACIONALES
}

La presente crisis económica internacional es la más seria y aguda desde el fin de la scgunda guerra mundial. Los cambios que experimentan ahora las economías capitalistas, y las significativas alteraciones que han experimentado las relaciones económicas internacionales, no son sino indicadores de esta profunda y severa crisis. No pueden éstas ser confundidas con fenómenos meramente circunstanciales, ni pueden ser rectificadas por soluciones de parche. No se puede retornar al status quo económico previo.

La crisis de los centros es de origen interno. Algunos lo atribuyen, o desearían atribuirlo, al mal funcionamiento de determinados sectores del sistema; a la declinación en la eficiencia marginal del capital; a la pérdida de la disciplina financiera, que a su vez alimenta el proceso inflacionario; a la excesiva centralización o concentración del mercado; a la disparidad entre la productividad agrícola e industrial, y así sucesivamente. Otros perciben estas contradicciones internas en forma más dramática: como determinantes del futuro del sistema. Lo que es fundamental, sin embargo, es que esos cambios internos que se efectúan en las economías centrales no responden sólo a contradicciones "económicas" internas, sino que reflejan también cambios cada día más acentuados de los valores en las propias sociedades industriales.

Tanto dentro de las sociedades desarrolladas como en aquellas en vias de desarrollo surgen hoy profundos interrogantes acerca del tipo de crecimiento logrado o al cual se aspira, y de las ambivalencias del progreso económico. Básicamente, este interrogante ha surgido a propósito de la búsqueda de la calidad del medio ambiente y de las extravagancias a las cuales está conduciendo la sociedad moderna

- Documento presentado en el Seminario sobre New Ways for the autonomous development of the Third World, rcalizado en Corfú, Grecia, en septiembre de 1975, bajo los auspicios del Conjunto Universitario Cándido Mendes, de Brasil. 
orientada a un consumismo desenfrenado. Esta perspectiva económica y social proporciona al momento histórico que vivimos una profundidad y dimensión virtualmente desconocidas en las crisis previas.

Estas crisis internas del actual sistema se proyectan en el comportamiento de las relaciones económicas internacionales. El mapa económico y político de la postguerra ha sufrido agudas mutilaciones. Ha habido una transición brusca desde un mundo unipolar a un mundo multipolar, primero con la emergencia del mundo socialista y ahora con la aparición - cada vez más cvidente- de la voz de los países del Tercer Mundo en las relaciones sociales y económicas del mundo moderno.

Los primeros síntomas de la crisis fueron señalados por el fracaso de las instituciones de postguerra, como las creadas en Bretton Woods, que llevó a nuestras naciones a un período de incertidumbre y contracción económica. La inseguridad económica se observa en las fluctuaciones del comercio internacional y de los actuales mercados cambiarios. El comportamiento errático de los grupos sociales en todo el mundo responde a la inseguridad básica de las relaciones sociales y comunitarias de la humanidad. Las muchas predicciones catastrofistas sobre el futuro de la humanidad confirman que se ha quebrado la fe tradicional en la ciencia como solución de todos los problemas.

A estas incertidumbres se ha añadido el estancamiento con inflación en los grandes centros, que si bien muestra algunos signos de disminución en los Estados Unidos, todavía persiste en otros países y amenaza con acelerarse en algunos donde se esperaban, al menos, signos de recuperación.

Se observa además en este panorama, que hasta los remedios económicos convencionales - correspondientes a un molde neoliberal- han fracasado o han respondido con desconocida lentitud. Cualquiera sea la hipótesis sobre el momento presente, debemos admitir que hemos entrado en un período de gran incertidumbre que no permite a nadie, en la actualidad, prever el resultado final, pero que presagia un período de mucho menor crecimiento en los centros, y sujeto a dolorosas fluctuaciones.

Dentro de esta perspectiva, el Tercer Mundo está sometido a dos clases de tensiones: aquellas provocadas por la aspiración de sus sociedades de perfeccionar y acelerar su proceso de crecimiento, y aquellas generadas por cl incierto, y a menudo injusto, escenario internacional.

Ha habido cambios en el Tercer Mundo. Particularmente en América Latina, aunque también en otras regiones, se han obtenido éxitos notables en el campo del crecimiento económico y en la solución de algunos problemas sociales, especialmente en favor de las crecientes clases medias. Pero los problemas fundamentales perma- 
necen, y fuertes presiones sociales que exigen cambios están surgiendo en muchas partes de nuestro planeta. La persistencia de grandes sectores de pobreza en el mundo, la creciente disparidad en la distribución del ingreso en el interior y entre los países, la aceleración de la dependencia y la vulnerabilidad de las economías, así como las graves fluctuaciones en la situación económica internacional, son todos motivos justificados de preocupación.

Muchos sienten que estos problemas sólo pueden ser resueltos a través de proyectos nacionales autónomos de desarrollo económico y social. Sin embargo, dada la actual situación internacional de creciente dependencia e inseguridad, estos proyectos nacionales presentan mucho más dificultades que en el pasado. Las "reglas del juego" han cambiado considerablemente y ha aumentado también el riesgo de que el Tercer Mundo se convierta más en espectador que en actor.

Esta incertidumbre ha creado un doble movimiento dentro del Tercer Mundo. Por un lado, hay un grupo de países que pide, con voces cada vez más fuertes, la construcción de un nuevo orden económico internacional. Muchos países en desarrollo han adoptado esta posición en reuniones internacionales recientes. Por otra parte, están aquellos -también entre los países en desarrollo- que han perdido la esperanza de conseguir un cambio sustancial en las "reglas del juego" de la economía internacional (debido principalmente a una injusta y desigual distribución del poder) y que están presionando para un desarrollo económico y social del Tercer Mundo que sea autosuficiente, autónomo y cada vez más independiente de las actuales relaciones internacionales con el mundo desarrollado.

Cualquiera sea el resultado de esta polémica, es importante que, de partida, toda la comunidad internacional revise el actual sistema de relaciones internacionales con un nuevo espíritu y una búsqueda creativa de alternativas de posibles soluciones de transacción. El Tercer Mundo no sólo debe estar presente en esta labor de revisión, sino que debe tener unidad interna que le permita asegurar un equilibrio adecuado en el diálogo internacional. Para asegurar esa unidad, el Tercer Mundo debe identificar los denominadores comunes que unen antes que los elementos que podrían dividirlos. Esto, por supuesto, es parte de la ambiciosa tarea comenzada por las Naciones Unidas con el propósito de crear un nuevo orden económico internacional.

LA CONSTRUCCIÓN DE UN NUEVO ORDEN ECONÓMICO INTERNACIONAL: UNA OPORTUNIDAD RENOVADA PARA LA JUSTICIA A UN NIVEL MUNDIAL

En los últimos años, la acción persistente del Tercer Mundo en las Naciones Unidas ha llevado a nuevas concepciones y nuevas metas 
en lo que se refiere al sistema de relaciones internacionales. Estas nuevas concepciones se encarnan en el llamado "nucvo orden cconómico internacional", del cual es elemento inherente el reconocimiento universal de la crisis del antiguo orden establecido, y la necesidad de un nuevo sistema de relaciones internacionales.

Es difícil describir o definir este nucvo orden económico internacional. La larga enunciación de las resoluciones de la Asamblea General ha oscurecido tal vez el sentido de los principios que las inspiraron. Podemos, sin embargo, especificar los principales componentes que las naciones del Tercer Mundo han identificado como objetivos:

a) Auténtico equilibrio en el ejercicio del poder económico en el mundo. Reconocimiento claro de que los países en desarrollo deben tener más voz y peso en la toma de decisiones en el plano internacional. Democratización de conductas y procedimientos en los mecanismos internacionales.

b) Reconocimiento de que no se podrá encontrar un sistema viable y justo de relaciones internacionales si no se avizora una solución al problema de los precios y mercados para las materias primas de los paises en desarrollo. Los precios y los mercados son la fuente esencial de estabilidad monetaria en los países en desarrollo.

c) Reconocimiento de la necesidad de una aproximación completamente diferente a la transferencia de recursos, que más que a motivaciones caritativas responda a obligaciones morales de la humanidad, al interés de lograr el equilibrio político de la comunidad internacional, y al propio interés económico del mundo en desarrollo.

d) La construcción de un sistema monetario internacional nuevo, no dirigido por un pequeño grupo de países, sino más bien por instituciones y normas de funcionamiento verdaderamente internacionales.

e) Modificación sustantiva de la presente distribución internacional de las oportunidades económicas y laborales, reconociendo en particular las posibilidades dinámicas del Tercer Mundo en el campo de la industrialización.

f) Avances considerables en el control de los "nuevos actores" que han aparecido en la escena internacional, y que representan tanto poder como progreso: la tecnología y las empresas transnacionales. 
Estos principios fundamentales proporcionan unidad y coherencia a la acción concertada de las naciones del Tercer Mundo, que en la última Asamblea General de las Naciones Unidas tuvieron respaldo de toda la comunidad internacional.

Cabe destacar que por primera vez, se ha aceptado en el ámbito internacional que el nuevo orden no puede basarse en avances individuales aislados, sino en múltiples avances en tantos frentes como sea posible abarcar.

La transferencia de recursos pierde todo su sentido si los paises no reciben precios justos y remunerativos por sus productos básicos. El endeudamiento no es ciertamente una solución. Sólo tiene sentido dentro del marco de un creciente volumen de producción y, por lo tanto, de mayores ingresos provenientes de las exportaciones. Cualquiera otra opción simplemente confunde el problema.

Todo sistema monetario que no considere los problemas del comercio o del endeudamiento externo está condenado a fallar en su objetivo global, como ha demostrado la experiencia de Bretton Woods.

Es de vital importancia entonces al considerar objetivos específicos, tener en cuenta el objetivo global de cualquier nuevo enfoque de las relaciones económicas internacionales. Sobre esta base pues, podremos hacer algunos comentarios relativos a la transferencia de recursos en el ámbito internacional.

\section{EL NUEVO ORDEN Y LA TRANSFERENCIA DE RECURSOS}

Entre los problemas conceptuales básicos de un nuevo orden económico internacional está el de la transferencia de recursos. Este problema ha sido estudiado a fondo por muchos representantes del Tercer Mundo, y las recientes reuniones del Fondo Monetario Internacional y del Banco Internacional de Reconstrucción y Fomento nos han proporcionado algunas indicaciones de los progresos que hasta ahora se han hecho. Y las publicaciones del Foro del Tercer Mundo sobre este tema han reunido en forma clara y concisa los puntos de vista de los países en desarrollo.

Cualquier definición de un nuevo sistema de ayuda debe partir de un análisis crítico del orden existente. Aunque tales análisis se han dado a conocer reiteradamente en el Tercer Mundo, vale la pena señalar aquí sus principales características:

a) El viejo sistema de ayuda estaba basado principalmente en motivaciones políticas, y la ayuda se consideraba un acto de caridad. No 
había alli una concepción clara de igualdad o de distribución justa de la riqueza a través cle la transferencia de recursos. En muchos casos, las transferencias se hacian a través de préstamos o donaciones encuadrados en la estrategia de la guerra fría, o para reforzar alianzas políticas y militares. En otros casos, la "ayuda" se daba a antiguas colonias para consolidar lazos históricos entre la periferia y las economías centrales (por ejemplo, con el fin de obtener materias primas, fuerza de trabajo y nuevos mercados para los centros). Sólo recientemente, y aunque no todos los países respondan a las mismas motivaciones, éstas comenzaron a oricntarse hacia una distribución más justi y equitativa de los recursos.

b) Las sumas convenidas y prometidas por los países industrializados a los países en desarrollo en diversas conferencias de la UNCTAD equivalian sólo al $0,7 \%$ de su producto nacional bruto. Muy pocos países realmente entregaron tal suma en 1974. De hecho, la ayuda total alcanzó sólo al 0,33\%, y los pronósticos del Banco Mundial indican que esta suma decrece y seguirá decreciendo progresivamente, a no ser que se adopten medidas concretas por parte de los países industrializados para corregir tal situación.

c) La ayuda financiera u otras formas de ayuda han estado atadas a muchas condiciones, particularmente algunas de naturaleza política que han sido denunciadas por los países en desarrollo. Gran parte de la ayuda ha estado también ligada a importaciones provenientes del país donante. En muy pocos casos ha sido suficientemente flexible o adecuada para satisfacer las necesidades reales de los países en desarrollo.

d) Entretanto, los países en desarrollo han acumulado grandes deudas que alcanzan a más de 120 mil millones de dólares y que, con las tasas de interés y servicio, han mermado grandemente las sumas obtenidas por los países del Tercer Mundo merced a sus exportaciones. En algunos países, se ha estimado que entre el 30 y el $40 \%$ del valor total de sus exportaciones ha debido destinarse al servicio de la deuda.

Estos y otros puntos muestran claramente la debilidad inherente de los esquemas actuales para la transferencia de recursos, y por lo tanto, la necesidad de un nuevo orden económico internacional en que pueda asentarse un nuevo tipo de relaciones entre los países desarrollados y en desarrollo en lo que toca a la transferencia de recursos. 


\section{CARACTERÍSTICAS DEL NUEVO ORDEN}

a) Cualquier nuevo orden debe comenzar con una nueva concepción de la transferencia de recursos desde los países ricos hacia los países pobres, y esta transferencia ya no puede ser considerada como un simple acto de caridad. Dado el abismo existente entre las naciones ricas y las pobres, así como la tremenda disparidad entre sus niveles de vida, la transferencia de recursos debe, primero y sobre todo, ser considerada como una obligación moral por parte de la humanidad. (Las naciones ricas podrían ver en esto una carga que cllas solas deberían soportar; sin embargo, considerarlo asi sería olvidar la contribución histórica que las nuevas naciones más pobres han hecho a la prosperidad de las ricas).

La transferencia de recursos desde los países ricos hacia los países pobres debe tambićn ser vista como un requisito fundamental y necesario para establecer y mantener la paz y la seguridad mundiales. Sin algo de paridad y justicia en el reparto de los recursos mundiales, los desposcídos no podrán ya tener interés en mantener un orden que durante tanto tiempo los ha privado de las necesidades básicas de la vida.

Por último, la transferencia de recursos debe ser aceptada como un componente importante del crecimiento económico de todos los paises. El estímulo a las potencialidades comerciales del Tercer Mundo no puede sino ayudar al desarrollo mundial del comercio y, por lo tanto, estimular las ya estancadas economías de las sociedades industrializadas.

b) Una meta clara, implícita en la transferencia de recursos, es la automaticidad. Al respecto, el Foro del Tercer Mundo ha dicho:

"Un elemento de automaticidad debe ser incluido en la futura transferencia de recursos desarrollando algunas fuentes de financiamiento internacionales (tales como derechos sobre la explotación comercial de bienes pertenecientes a la comunidad internacional, como el lecho marino y el espacio); vinculando la creación de una nueva moneda internacional con la provisión de financiamiento para el desarrollo, y estableciendo algunas formas de impuestos internacionales, tales como una tasa sobre los recursos no renovables, los contaminantes internacionales y los gastos en armamentos".

c) Aún más, las nuevas relaciones deberian asegurar que la trans- 
ferencia de recursos no interfiera con los modelos de desarrollo adoptados por los países receptores, ni afecte las prioridades establecidas por ellos o por los estilos de vida adoptados. Una vez más, el Foro del Tercer Mundo ha destacado que:

"El concepto de la transferencia de recursos reales debería excluir todas aquellas transferencias que sean incompatibles con las prioridades nacionales de desarrollo. Por ejemplo, tal concepto debería excluir los bienes de consumo, las ventas militares o las transferencias de productos alimenticios (excepto en emergencias) que alteren los planes de producción de alimentos a largo plazo dentro del Tercer Mundo. Igualmente, la transferencia tecnológica debería promover la capacidad tecnológica, en lugar de perpetuar la dependencia tecnológica. El Tercer Mundo haría bien en rechazar todas aquellas transferencias de recursos que no contribuyan a sus objetivos de desarrollo a largo plazo".

d) Como corolario de lo anterior, el nuevo orden económico internacional debe prestar atención preferente a las necesidades básicas mínimas de los sectores más pobres de la humanidad, y a los programas destinados a aliviar sus sufrimientos. Al respecto, el Dr. Mahbub ul Haq expresó que:

"Esto proporcionaría a la vez un punto focal y una orientación al esfuerzo de ayuda internacional, y lo haria por un periodo limitado, hasta que algunas de las peores manifestaciones de la pobreza -desnutrición, analfabetismo y condiciones de vida inhumanas- sean superadas a través del esfuerzo internacional, y de la capacidad cada vez mayor de los gobiernos nacionales para lanzar un ataque directo a la pobreza. Estos programas, sin embargo, no deberían basarse en una simple transferencia de ingresos a los pobres - que crearía una dependencia permanentesino en el aumento de la productividad de los pobres y en su incorporación al sistema económico. Es difícil estimar cuánto habría que invertir para que la mayor parte de la humanidad pueda satisfacer las necesidades humanas mínimas: para ello se necesita mucho trabajo conceptual y empírico (como el que llevan a cabo actualmente la fundación Dag Hammarskjold y el Banco Mundial) . No obstante una estimación aproximada basada en diversos estudios del Banco Mundial, señala que se requeriría una inversión total de aproximadamente 125 mil millones de dólares (a precios de 1974) en un período de 10 años (por ejemplo 42 mil millones en alimentación y nutrición, 25 mil millones en educación, 28 mil millones en suministro de agua rural y urbana, 
16 mil millones en vivienda urbana, 8 mil millones en transporte urbano y 6 mil millones de dólares en población y programas de salud). Por supuesto estas estimaciones variarán considerablemente según el estilo de desarrollo buscado por diversos países. Sin embargo, las ventajas de establecer tal meta, para la eliminación de la pobreza es que ésta puede ser fácilmente comprendida por el público ( $\mathrm{y}$, ojalá, por los políticos) en las naciones ricas; puede ser objeto de un esfuerzo compartido entre los gobiernos nacionales y la comunidad internacional; proporciona una fórmula para la distribución de la ayuda concesional, y permite fijar un período específico de tiempo en el cual la tarea debe ser completada".

e) En materia de asistencia, la redefinición de un nuevo orden debe encontrar también una solución al problema de la vasta e inmanejable deuda que han acumulado los países en desarrollo. En la actualidad, la ayuda financiera se presta en términos tan onerosos que alrededor del $50 \%$ de todas las nuevas transferencias corresponden al servicio de antiguas deudas. En los foros de la UNCTAD se ha sugerido la conveniencia de convocar a una conferencia sobre renegociación de la deuda externa que tendría lugar a más tardar hacia fines de 1976. Los objetivos de esta conferencia serían los de proporcionar a los países en desarrollo opciones adecuadas y máxima flexibilidad no sólo para amortizar sus deudas, sino para permitirles enfrentar las variadas situaciones que se dan en sus respectivos países.

f) Finalmente, y relacionado con lo anterior, la comunidad internacional debe prestar atención concertada a la creación de un código de conducta que reglamente las relaciones entre los países en desarrollo y las empresas transnacionales, así como todas las formas de inversión privada extranjera. La reglamentación de esta forma de actividad es de fundamental importancia para los países del Tercer Mundo.

Todo lo expresado anteriormente sobre el nuevo sistema de ayuda se basa en los fundamentos conceptuales y los principios que llamaron a crear un orden internacional totalmente nuevo. La transferencia de recursos debe tener un efecto correctivo en la situación actual de los países en desarrollo. Los países más ricos y seguros en el aspecto financiero deben estar dispuestos a ayudar más adecuadamente a los miembros menos afortunados de la comunidad internacional y a reconocer las dificultades especiales que confrontan muchos de los países del Tercer Mundo. Este reconocimiento es de fundamental importancia tanto para el concepto del nuevo sistema de ayuda, como para el nuevo orden económico internacional. 


\section{LA COOPERACIÓN FINANCIERA Y LA TRANSFERENCIA} DE RECURSOS ENTRE LOS PAÍSES EN DESARROLLO

Los capítulos precedentes tienen en cuenta dos conclusiones básicas: a) el nuevo orden económico internacional no puede construirse sobre las líneas deseadas, si no hay un avance simultáneo en varios frentes, uno de los cuales es la transferencia de recursos, y otro igualmente importante es el comercio bajo condiciones justas; b) todo nuevo concepto sobre la transferencia de recursos reales debería basarse en una cooperación real entre las naciones, que reconozca la obligación moral de ayuda y que rechace la noción falsa de la ayuda como caridad o como instrumento de manipulación política.

Ultimamente hemos sido testigos de un nuevo fenómeno en el plano internacional: la abundancia de recursos financieros de los paises exportadores de petrólco. Estos países, que sólo en los últimos años han obtenido grandes ganancias netas y acumulado reservas monetarias, pueden hacer inversiones significativas en el extranjero.

Este fenómeno ha dado lugar a una controversia internacional acerca de cómo tales países exportadores de petróleo pueden y deben colaborar con otras naciones del Tercer Mundo con miras a resolver problemas de balance de pagos. Pese a la variedad de estilos y procedimientos, la cooperación política inicial y la alianza de la OPEP con el Tercer Mundo deben reconocerse como particularmente importantes.

¿Qué papel podrían desempeñar los países de la OPEP dentro del marco de las ideas anteriormente expuestas sobre nuevas formas de cooperación internacional? ¿Qué podrían hacer los países en desarrolo para aumentar su solvencia y para promover las oportunidades de inversión en proyectos de desarrollo en sus respectivos países?

El análisis de las interrogantes antes planteadas debería comenzar por configurar un panorama de las diversas "realidades" de los países de la OPEP, realidades que, y esto debe entenderse desde la partida, son muy importantes para elaborar un concepto de la planificación del desarrollo a largo plazo.

a) Ante todo, parece que en los países de la OPEP Ios recursos petroquímicos actuales y potenciales no se encuentran distribuidos en forma equitativa. De acuerdo con las estimaciones del Banco Mundial, algunos de los paises de la OPEP tienen recursos bastante limitados y por tanto se prevé su agotamiento o disminución en un futuro próximo. Otros, en cambio, cuentan con recursos a mayor plazo y 
pueden por tanto acumular riqueza. Sin embargo, muchos de estos países confrontan problemas de subdesarrollo interno y para superarlos deberán invertir importantes sumas de dinero. Por este motivo, es posible que su balance de pagos no sea negativo, pero como tienen que importar mano de obra especializada, equipos y tecnología, los fondos sobrantes para inversiones en el extranjero pueden ser bastante limitados.

Por lo tanto, al calcular los recursos potenciales de que puedan disponer las fuentes de la OPEP hay que ser realistas y tener en cuenta la situación relativa de los diversos países que la integran.

b) Los países de la OPEP no son ricos. Sus necesidades para el desarrollo son y seguirán siendo sustanciales. Naturalmente, esto hace que se muestren renuentes a invertir en áreas inseguras de inversión financiera. El deseo de garantizar la seguridad financiera y la recuperación de las inversiones es enteramente comprensible, puesto que sus recursos no son renovables.

c) Las posibilidades de inversión en los países del Tercer Mundo difieren mucho y lo mismo sucede con su capacidad de amortizar tales inversiones. De acuerdo con los procedimientos tradicionales para el otorgamiento de préstamos, es natural que las inversiones se hagan en aquellos sectores en que haya cierta garantía de seguridad y de rentabilidad. Por esta razón los paises en vías de desarrollo más prósperos y más desarrollados tienden a atraer más inversiones extranjeras. Sin embargo, los países en desarrollo más pobres son los que más necesitan la inversión de capitales externos. Por otra parte, la inversión en las sociedades en desarrollo es algo mucho más complejo de lo que ha sido tradicionalmente en las economias desarrolladas. Por estas y otras razones los países de la OPEP se han inclinado manifiestamente a concentrar sus inversiones sólo en algunas naciones del Tercer Mundo.

d) Finalmente, también cabe observar que, por las razones ya mencionadas y por la falta de suficientes programas de inversión de los países en desarrollo, los países de la OPEP han concentrado sus inversiones en diversas áreas de los países industrializados que ofrecen utilidades seguras, y han destinado una fuerte porción de sus nuevos ingresos a su propio desarrollo. En última instancia, y es bueno anotarlo, la mayoría de los desembolsos se han destinado a comprar bienes que sólo se producen en las sociedades industrializadas, con lo cual han contribuido en forma dinámica a la recuperación de los Grandes Centros. 
Los países en desarrollo también presentan realidades diferentes. En primer término, están los más pobres, los más desposeídos del Tercer Mundo. Las circunstancias y necesidades de estos paises son tales que la ayuda que requieren para su desarrollo es cuantiosa y debe prestarse en condiciones concesionales.

En el caso de los países más pobres que, de acuerdo con las cifras del Banco Mundial, albergan más de mil millones de personas, la transferencia de recursos no sólo debe efectuarse en términos concesionales, sino también, y de manera especial, debe consistir en donaciones para alimentos.

Otros países, como muchos de Amćrica Latina, cuyo nivel de desarrollo es algo más alto, sólo pueden aspirar a la cooperación en términos más convencionales, a una participación más activa en los mercados de capital y en los mercados comerciales de las naciones más industrializadas.

La existencia de los extremos arriba mencionados plantea un problema para la utilización de los recursos de la OPEP. La última forma representa por cierto una inversión más segura, pero la primera constituye una fuerte obligación moral. Se requerirá gran imaginación y espíritu creador así como solidaridad entre los paises del Tercer Mundo, para conciliar las diferencias y utilizar adecuadamente los fondos que proporcionen los países de la OPEP.

Es efectivo que hay muchos países del Tercer Mundo que todavía no definen claramente sus modelos o estrategias de desarrollo; por otra parte, no todos tienen un claro concepto de la clase de proyectos que desean llevar a cabo para construir su futuro económico y social. La capacidad de atraer recursos que poseen estos países es por lo tanto débil y la solución de sus problemas más difícil.

Bien sabemos que la falta de proyectos de inversión remunerativos y financiables dentro del Tercer Mundo, unida a un criterio inadecuado para la transferencia de recursos, hacen extremadamente difícil que sea sano el proceso de crecimiento. Esto debe reconocerse como una de las muchas realidades que obstaculizan el desarrollo de la región.

Pese a lo anterior, los países en desarrollo no parten de la nada. Muchas naciones del Tercer Mundo han comenzado a colaborar política y económicamente con los países cle la OPEP y como consecuencia de ello han cambiado sustancialmente las condiciones existentes. Cabe destacar la creciente participación de los fondos de la OPEP en recursos multilaterales, como los del Fondo Monetario Internacional, el Banco Mundial y los bancos regionales de desarrollo, como también la asistencia directa de la OPEP a los países importadores de petróleo del Tercer Mundo a través de préstamos en condi- 
ciones concesionales y otras ayudas financieras destinadas a aliviar problemas de balance de pagos.

TEMARIO PARA EL EXAMEN DE UN MARCO DE COOPERACIÓN Y TRANSFERENCIA DE RECURSOS ENTRE LA OPEP Y OTROS PAÍSES DEL TERCER MUNDO

Tras las consideraciones anteriores, cabría señalar algunos puntos que podrían examinarse relativos a un programa de cooperación, con especial referencia a la transferencia de recursos, entre los países de la OPEP y otros países del Tercer Mundo.

1) Hay que reconocer de partida que la transferencia de recursos es sobre todo una cuestión política y no un simple problema técnico o económico. Esto significa que debe existir, o debe crearse la voluntad política en los países de la OPEP de iniciar nuevas formas de cooperación con los países del Tercer Mundo. Sin esta disposición o voluntad política no será posible una nueva aproximación al problema de la transferencia de recursos.

2) Debe fortalecerse también la solvencia de otros países en desarrollo para que puedan atraer las inversiones de la OPEP. Los proyectos o programas de desarrollo deben ser sólidos, y garantizar a las fuentes que proporcionan los fondos de la OPEP que se realizará un intento scrio, no sólo de utilizar adecuadamente esos nuevos recursos, sino de reembolsarlos en un plazo aceptable para ambos.

3) Un programa ampliado de cooperación podria incluir los siguientes campos:

a) Apoyo a los problemas de balance de pagos originados por el alza de los precios del petróleo. Este tipo de apoyo se ha iniciado ya a través de programas de préstamos tanto a corto como a largo plazo. La fórmula concertada entre Venezuela y América Central representa un esfuerzo scriero en este sentido. De acuerdo con esa importante iniciativa, los paises centroamericanos pueden pagar una parte de sus compras de petrólco en su propia moneda y a mediano plazo. Esto contribuye de manera importante a resolver sus problemas de balance de pagos, ya que les proporciona apreciable acceso a préstamos en condiciones concesionales para adquirir el petrólco que necesitan. Otras naciones exportadoras de petróleo deberían estudiar seriamente esta proposición, que modifica de manera concreta y constructiva 
procedimientos anteriores. Esta iniciativa ofrece la posibilidad tanto de ayudar a satisfacer las necesidades de los países en desarrollo como de garantizar una verdadera recirculación (o reciclaje) de recursos entre estos mismos países. No debería descartarse la creación de un fondo para el desarrollo, con parte de los recursos provenientes de futuras alzas de los precios del petróleo. Este fondo permitiría adquirir productos petroquímicos y otros rubros necesarios, como alimentos y tecnología, que muchos países en desarrollo requieren en forma apremiante y que podrían provenir de los propios países del Tercer Mundo.

b) Apoyo a la cooperación financiera multilateral entre los países del Tercer Mundo. Quizá no sea ésta la oportunidad apropiada para mencionar las contribuciones actuales de los países de la OPEP al Fondo Monetario, al Banco Mundial y a los diversos bancos de desarrollo regional. Sin embargo, cabe recordar aquí que las inversiones que han estado haciendo últimamente estas instituciones en los países en desarrollo desempeñan una importante función y merecen el continuado apoyo de los países de la OPEP en la medida en que tales recursos fluyan directamente hacia los países en desarrollo afectados por crisis de balance de pagos.

c) Apoyo a las iniciativas sobre cooperación financiera en el campo del comercio entre los países del Tercer Mundo. En este momento, es de especial interés para los países en desarrollo la posibilidad de establecer un banco de productos básicos. Esta iniciativa se está estudiando en el sistema de las Naciones Unidas, particularmente en el Grupo de los 77. Dejando de lado las soluciones ofrecidas, el concepto de un banco de productos básicos de esta naturaleza deberia ser de gran interés para las fuentes financieras de la OPEP. Por otra parte, contribuiría a regular los precios de las materias primas en todos los países del Tercer Mundo.

d) Apoyo a programas transitorios de inversión en los países en desarrollo. Teniendo en cuenta las reservas antes señaladas, hay que aceptar y utilizar alguna forma de ayuda bilateral a corto plazo para las necesidades de desarrollo de los países del Tercer Mundo. Las donaciones y los préstamos en condiciones favorables podrían utilizarse de inmediato para proyectos de esta naturaleza.

4) Las opciones precedentes se han estado investigando. La creación de otras modalidades o metodologías está sujeta a la voluntad política y a la inventiva de donantes y receptores. Para el solo efecto 
de examinarlas en la presente reunión se ofrecen las siguientes sugerencias:

a) Apoyar la expansión de los mercados de los países del Tercer Mundo en los países de la OPEP. Desde el primer momento ha sido evidente que muchos países de la OPEP han estado consumiendo, y más bien sobreconsumiento, productos disponibles en el mundo occidental. Lo que aún no se investiga a cabalidad es la importación de bienes de consumo, principalmente de materias primas para alimentos y fertilizantes desde los países en desarrollo. En muchos casos los países de la OPEP tienen déficit de alimentos y, por lo tanto, bien podrían considerar la posibilidad de realizar programas de inversión en industrias que hacen uso intensivo de capital en otros países del Tercer Mundo, con el fin de aumentar la capacidad de éstos para producir y exportar alimentos. Sería fácil suscribir acuerdos sobre inversiones y exportaciones entre estos países.

b) Apoyar inversiones en exploración y aprovechamiento de los recursos naturales, particularmente en materia de abastecimiento de energía. Muchos países en desarrollo tienen grandes recursos energéticos potenciales cuyo aprovechamiento requiere inversiones externas. A menudo, tales fuentes de energía atraen a grandes empresas transnacionales, pero por razones bien conocidas, algunos países del Tercer Mundo se muestran cada vez más renuentes a comprometerse con estas empresas en ciertos campos muy delicados desde el punto de vista político. Sin embargo, tal vez pueda idearse un nuevo tipo de inversiones a través del cual los paises de la OPEP puedan destinar fondos a la exploración de recursos y recibir a cambio remuneración en especie -en otros productos de los países en desarrolloy a precios justos y razonables en función del mercado internacional.

c) Prestar apoyo a programas multinacionales en el plano regional y subregional. Muchas modalidades de inversión podrían ser de gran atractivo para las fuentes financieras de la OPEP si se dieran en el plano regional o subregional. Incluso las enormes empresas transnacionales han reconocido el valor de tales modalidades más amplias de inversión potencial, y por ello los países latinoamericanos resolvieron últimamente elaborar su propio tipo de empresa transnacional intergubernamental que les permita aprovechar mejor sus potencialidades.

d) Apoyar el desarrollo de las potencialidades tecnológicas del Tercer Mundo. El Tercer Mundo, o los países en desarrollo, poseen 
grandes recursos humanos y potencialidades científicas y tecnológicas, hasta ahora subutilizadas, particularmente para confrontar problemas internos de desarrollo. La subutilización ha sido en parte un problema de determinación política y en parte, un problema de fuentes de recursos. Por ambos motivos puede preverse la utilización de esta potencialidad por los países del Tercer Mundo. Los países de la OPEP bien podrían invertir sus recursos en la creación de un nuevo "banco tecnológico".

5) Las acciones decisivas por parte de los países en desarrollo deberían ejecutarse de manera de poder materializar las anteriores concepciones.

Los elementos fundamentales de esta acción deberían ser la definición del modelo o estrategia de desarrollo requerido y una concepción de la forma en que se distribuirán los distintos recursos del país, como es natural, teniendo presentes las necesidades de sus grandes mayorias. También hay que considerar las fuerzas del mercado, porque son una realidad del mundo en que vivimos, pero ello no debe impedirnos descubrir nuevas fucrzas que permitan a los países en desarrollo explotar y compartir sus recursos más efectivamente unidos. Los proyectos de desarrollo, sean de origen nacional o regional, pueden elaborarse sobre una base firme $y$, sin duda, pueden atraer las inversiones necesarias para su ejecución.

Otro factor que no hay que olvidar es que son los propio países en desarrollo quienes deben buscar soluciones al problema de la deuda externa acumulada. La solvencia de muchos países se ha determinado por su capacidad de pagar deudas anteriores. Aunque para muchos esto puede parecer injusto, hay que comprender que la capacidad de atraer capitales aún se basa en la solvencia. Por lo tanto, hay que buscar soluciones para establecer o restablecer el prestigio financiero de los paises o regiones del Tercer Mundo.

En tercer lugar, los países en desarrollo deben elaborar e investigar más a fondo los mecanismos institucionales que facilitarían las inversiones provenientes de la OPEP y de otras fuentes. En esta investigación es de primordial importancia elaborar un código de conducta para las actividades de las empresas transnacionales y para las actividades multinacionales, de manera que los acuerclos contengan ciertas garantías tanto para el inversor como para las naciones receptoras. La creación de tal "código de conducti" apremia en bien del clesarrollo y de las estrategias del Tercer Mundo. 